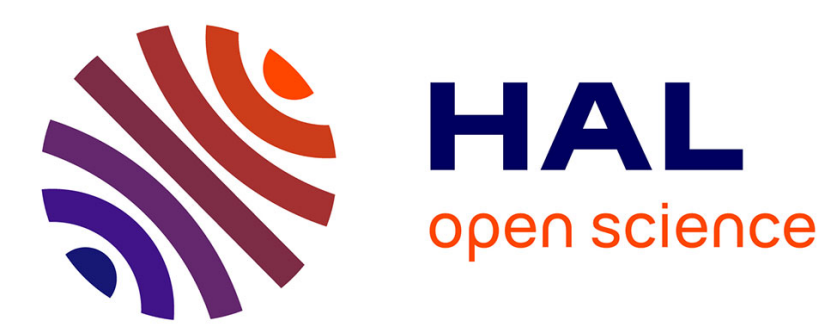

\title{
Tank-treading of microcapsules in shear flow
}

Clément de Loubens, Julien Deschamps, Florence Edwards-Lévy, Marc

Leonetti

\section{To cite this version:}

Clément de Loubens, Julien Deschamps, Florence Edwards-Lévy, Marc Leonetti. Tank-treading of microcapsules in shear flow. Journal of Fluid Mechanics, 2016, 789, pp.750-767. 10.1017/jfm.2015.758 . hal-02020215

\section{HAL Id: hal-02020215 https://hal.science/hal-02020215}

Submitted on 5 Nov 2019

HAL is a multi-disciplinary open access archive for the deposit and dissemination of scientific research documents, whether they are published or not. The documents may come from teaching and research institutions in France or abroad, or from public or private research centers.
L'archive ouverte pluridisciplinaire HAL, est destinée au dépôt et à la diffusion de documents scientifiques de niveau recherche, publiés ou non, émanant des établissements d'enseignement et de recherche français ou étrangers, des laboratoires publics ou privés. 


\title{
Tank-treading of microcapsules in shear flow
}

\author{
C. de Loubens ${ }^{1,2}$, J. Deschamps ${ }^{1}$, F. Edwards-Levy ${ }^{3}$ and M. Leonetti ${ }^{1}$ \\ $\dagger$ \\ ${ }^{1}$ Aix-Marseille Université, CNRS, Centrale Marseille, IRPHE UMR 7342, Marseille, France \\ ${ }^{2}$ LIPhy, CNRS-UMR 5588, Université de Grenoble I, 38402 Saint Martin d'Hères France \\ ${ }^{3}$ Faculté de Pharmacie, Université de Reims Champagne-Ardenne, CNRS, ICMR UMR 7312, \\ Reims, France
}

(Received 5 November 2019)

We investigated experimentally the deformation of soft microcapsules and the dynamics of their membrane in simple shear flows. Firstly, the tank-treading motion, i.e. the rotation of the membrane, was visualized and quantified by tracking particles included in the membrane by a new protocol. The period of membrane rotation increased quadratically with the extension of the large axis. The tracking of the distance between two close microparticles showed membrane contraction at the tips and stretching on the sides, a specific property of soft particles such as capsules. Present experimental results are discussed in regard to previous numerical simulations. This analysis showed that the variation of the tank-treading period with the Taylor parameter (deformation) cannot be explained by purely elastic membrane models. It suggests a strong effect of membrane viscosity whose order of magnitude is determined. Secondly, two distinct shapes of sheared microcapsules were observed. For moderate deformations, the shape was a steady ellipsoid in the shear plane. For larger deformations, the capsule became asymmetric and presented a S-like shape. When the viscous shear stress increased by three orders of magnitude, the small axis decreased by $70 \%$ whereas the large axis increased by $100 \%$ before any break-up. The inclination angle decreased from $40^{\circ}$ to $8^{\circ}$, almost aligned with the flow direction as expected by theory/numerics on capsules and experiments/theory/numerics on drops and vesicles. Whatever the microcapsule size and the concentration of proteins, the characteristic lengths of the shape, the Taylor parameter and the inclination angle satisfy master curves versus the longest axis or the normalized shear stress or the capillary number in agreement with theory for non negligible membrane viscosity in the regime of moderate deformations. Finally, we observed that very small deviation from sphericity gave rise to swinging motion, i.e. shape oscillations, in the small deformation regime. In conclusion, this study of tank-treading motion supports the role of membrane viscosity on the dynamics of microcapsules in shear flow by both independent methods which compare experimental data with numerical results in the regime of large deformations and with the theory in the regime of moderate deformations.

\section{Introduction}

Knowledge of microcapsule dynamics is important in order to design the encapsulation of drugs and control their delivery in numerous applications. These objects, liquid drops bounded by a membrane, have specific properties used to control the delivery of drugs in medicine and biology (Hejazi \& Amiji (2003)) or specific molecules dedicated to cosmetics or nutrition (Shewan \& Stokes (2013)). This control can be achieved by

$\dagger$ Email address for correspondence: marc.leonetti@univ-grenoble-alpes.fr, clement.de-loubens@univ-grenoble-alpes.fr 
passive diffusion through the membrane or by external active stimuli ( $\mathrm{pH}$, temperature, ultrasounds) which lead to burst or mechanical stresses inducing cyclic deformations (Long et al. (2015)). There is also a growing interest to study capsules and vesicles under flow to have a deeper understanding of the dynamics of red blood cells (RBCs) in vessels and microfluidic biochips. Indeed, each of these soft particles share with RBCs specific mechanical properties that can be tuned independently (Vlahovska et al. (2009), Barthès-Biesel (2011), Li et al. (2013), Freund (2014)).

According to the constitutive material of the membrane different mechanical properties and dynamics in flow are obtained. The membrane of a vesicle is a two-dimensional fluid incompressible lipid bilayer with a resistance to bending, no membrane shear elasticity and a negligible membrane viscosity (Abreu et al. (2014)). The membrane of a capsule is a thin elastic solid made of a complex network of cross-linked polymers. The thickness and the degree of cross-linking can be tuned by the process parameters (Andry et al. (1996), Gunes et al. (2011)) to control the shear elasticity and the area dilation resistance. For example, capsules made of an artificial polymer such as polysiloxane or nylon present permanent deformations and break for small or moderate deformations (up to $50 \%$ of stretching, Chang \& Olbricht (1993a), Koleva \& Rehage (2012)). On the contrary, membranes made of cross-linked proteins such as human serum albumin (HSA) can sustain up to $180 \%$ of stretching without plastic deformations and break-up (de Loubens et al. (2015)).

In flow, capsules and vesicles present a diversity of shapes and dynamics, revealed by experiments, numerical simulations and theoretical investigations. The configurations of flow are often similar but the salient ingredients to understand deeply the dynamics are different: on one hand, the bending modulus and the vesicle deflation and on the other hand, the shear and dilation elastic moduli and the reference state (in the resting state) which is like a memory shape (Kessler et al. (2008)). In fact, beyond these basic elements, the mechanical characteristics of capsule membranes can be more complex with contributions of bending resistance to stabilize localized shape modulations, namely wrinkles (Walter et al. (2001), Koleva \& Rehage (2012)) and membrane viscous dissipation (Yazdani \& Bagchi (2013), Barthes-Biesel \& Sgaier (1985)) which is particularly relevant in RBCs for example (Nash \& Meiselman (1983), Hochmuth et al. (1979)). This wealth of membrane properties makes the study of capsules very attractive to determine accurately the role of the different physical parameters in the global dynamics. Indeed, the shapes and dynamics of capsules in flow have been extensively studied numerically and theoretically in several configurations: simple shear flow (Lac et al. (2004), Walter et al. (2010), Omori et al. (2012), Sui et al. (2008), Bagchi \& Kalluri (2009)), elongation flow (Dodson \& Dimitrakopoulos (2009)), in capillaries (Lefebvre et al. (2008)) or in a corner (Zhu \& Brandt (2015)). This knowledge allows to develop new methods to measure the elastic parameters using either the results of the linear theory of deformations as in the seminal work of Chang \& Olbricht (1993b) or numerical simulations to develop inverse methods (Lefebvre et al. (2008), de Loubens et al. (2015)).

Compared to the numerous numerical studies, the experimental investigations of capsule shapes and dynamics in flow are scarce and often limited to the regime of moderate deformations due to the capsule breakup: capsules in shear flow (Chang \& Olbricht (1993a), Walter et al. (2001)), in elongation flow (Chang \& Olbricht (1993b)) and in capillaries (Risso et al. (2006)). Considering the tank-treading motion, probably the main dynamics of soft particles in shear flow, the rotation of the membrane has only been visualized and quantified by tracking fluorescent beads or particles attached to the membrane of vesicles (Kantsler \& Steinberg (2005)) and RBCs (Fisher et al. (1978), Abkarian et al. (2007)). 


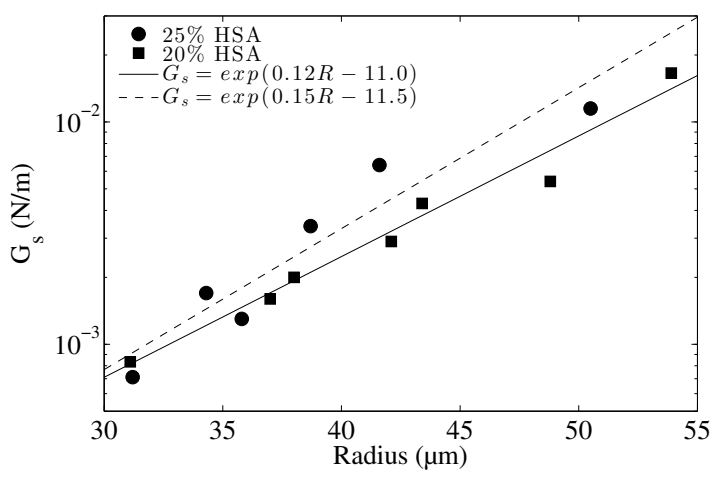

Figure 1: Shear elastic modulus $G_{s}$ as a function of the capsule radius $R$. Values were determined in elongation flow experiments (see de Loubens et al. (2014) for details). Data are fitted with exponential laws for each HSA concentration.

In this paper, we investigate experimentally the deformation, the orientation and the motion of the membrane of microcapsules in shear flow up to large deformations (variation of the longest axis up to $100 \%$ ). In section 2, the techniques used to decorate the membrane of HSA microcapsules with particles and the design of the shear flow chamber are presented. In section 3, the motion of capsules in shear flow is fully characterized: rotation of the membrane, period measurement, evolution of the shapes on three decades of shear stress. To highlight the role of membrane viscosity and determine its value, two complementary approaches are used: comparisons between experimental data with numerical simulations of Yazdani \& Bagchi (2013) in the regime of large deformation and with the theory of Barthes-Biesel \& Sgaier (1985) in the regime of moderate deformations.

\section{Methods}

\subsection{Microcapsules preparation}

Human serum albumin (HSA) was provided by LFB Biomedicaments. The organic solvents were from SDF, terephthaloyl chloride from Acros Organics, and surfactants (sorbitan trioleate, polysorbate) from Sigma. The yellow iron oxide powder was a gift from BASF. Batches of cross-linked HSA microcapsules were prepared using the interfacial cross-linking method (Andry et al. (1996), de Loubens et al. (2014)), using terephthaloyl chloride as cross-linker. Briefly, a 20 or $25 \mathrm{~g} / 100 \mathrm{~mL}$ HSA solution was prepared in a pH8 phosphate buffer. This aqueous solution was emulsified in cyclohexane containing $2 \mathrm{~g} / 100 \mathrm{~mL}$ sorbitan trioleate at a stirring speed of $1700 \mathrm{rpm}$. A $2.5 \mathrm{~g} / 100 \mathrm{~mL}$ solution of terephthaloyl chloride in chloroform:cyclohexane (1:4 volume / volume) was then added to the emulsion and the cross-linking reaction was allowed to develop for $30 \mathrm{~min}$. The reaction was stopped by dilution of the reaction medium. The microcapsules were separated from the organic phase by centrifugation, and washed successively with cyclohexane, with water containing $2 \mathrm{~g} / 100 \mathrm{~mL}$ polysorbate and finally thrice with pure water.

In order to include visible granulations on the membrane to characterize its tanktreading motion, the same procedure was performed with an additional step, consisting in dispersing $1 \mathrm{~g} / 100 \mathrm{~mL}$ iron oxide powder of typical size 1-2 $\mu \mathrm{m}$ in the HSA buffered solution prior to the emulsification step. The resulting particles looked ochre yellow to the naked eye, whereas their granulations made them appear opaque black using optical 
microscopy. We had initially checked mixing a little droplet of capsules in water with a larger one with glycerol that capsules flattened up to a pancake shape due to the exit of water and after inflated with glycerol entry. Note that this also highlighted the difference of permeabilities of the membrane to water and glycerol. Finally, the capsules were stirred in a large volume of $98 \%$ glycerol (VWR) during one night, in order to allow the internal medium of each capsule to equilibrate with the external one by diffusion through the pores of their membrane. Thus, the fluid was then the same inside and outside of the capsule.

\subsection{Elastic properties of microcapsules}

The emulsification step in the preparation procedure generates capsules with some size dispersion. The size distribution of the resulting capsules was measured by laser diffraction (Malvern Mastersizer 2000). The median radius was $39 \mu \mathrm{m}$. Ninety and ten percent of the distribution lied below 54 and $28 \mu \mathrm{m}$, respectively.

These microcapsules have already been mechanically characterised in elongation flows (see de Loubens et al. $(2014,2015)$ for details). We summarize in this section the main conclusions useful for the present paper. Firstly, we have shown that the elastic shear modulus $G_{s}$ of the resulting capsules increases of several orders of magnitude when the HSA concentration and the capsule size increase. A simple material balance applied between membrane HSA and volumic available HSA before cross-linking explains the qualitative variation with the size and HSA concentration (de Loubens et al. (2014)). Secondly, the study of these capsules in large deformations (i.e. up to $180 \%$ of stretching of the largest axis) allowed us to determine the bidimensional constitutive law of their membrane. We concluded that the membrane of HSA capsules obeys to the generalized Hooke law with a surface Poisson ratio $\nu_{s}$ of 0.4. Thus, the area dilation modulus $K=$ $G_{s}\left(1+\nu_{s}\right) /\left(1-\nu_{s}\right) \approx 2.3 G_{s}$ varying on several orders of magnitude with the microcapsule size and the initial concentration of HSA. The constitutive law provides a relationship between the tension tensor and the stretch ratio or principal stretches in the direction $j$, $\lambda_{j}$ calculated versus the reference state. The principal tension $T_{1}$ is given in the following, $T_{2}$ is obtained by switching the indices 1 and 2 :

$$
T_{1}=\frac{G_{s}}{1-\nu_{s}}\left[\lambda_{1}^{2}-1+\nu_{s}\left(\lambda_{2}^{2}-1\right)\right]
$$

The evolution of the elastic shear modulus $G_{s}$ with the radius $R$ was determined in de Loubens et al. (2015) (figure 1). With the exponential fits of these data, we can determine a value of $G_{s}$ for each capsule studied in the present paper. Finally, a total of twenty capsules were studied with a radius ranging from 20 to $68 \mu \mathrm{m}$. The polydispersity of the microcapsules which is a consequence of the classic protocol of interfacial polymerization of an emulsion is in fact a chance allowing to vary on several orders of magnitude the normalized shear stress and the capillary number as shown later.

\subsection{Shear flow}

Capsules were studied in a $1 \mathrm{~mm} \times 100 \mathrm{~mm}$ rectangular channel of $5 \mathrm{~mm}$ depth (figure 2). The channel was made of opaque PVC to minimise light reflection near the boundaries. Capsules were visualized from the side of the channel in the $z$ direction. The fluid was injected by a glass syringe pushed by a PI actuator M235-52S. The outlet was at ambient pressure. The channel was mounted on a microscope Olympus IX-71 with magnification x10 or x20 (1.701 or $0.851 \mu \mathrm{m} / \mathrm{pix})$. A high speed camera Photron Fastcam SA3 recorded up to 4000 frames per second.

We defined a region of interest (ROI: $200 \mu \mathrm{m} \times 1 \mathrm{~mm} \times 1 \mathrm{~mm}$ ) in the channel located at the middle of its two largest lengths (in $x, z$ directions) and at the wall of its smallest 


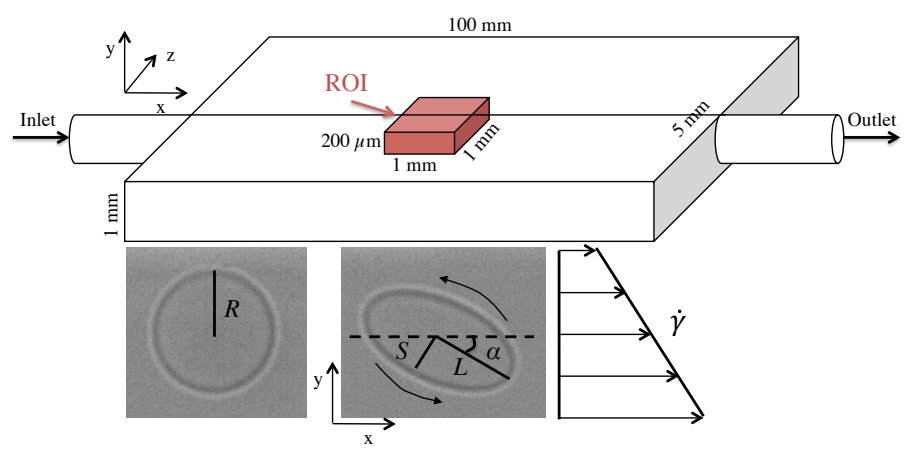

Figure 2: Experimental set-up. A spherical capsule of radius $R$ is deformed by shear flow in a region of interest (ROI) where the shear rate $\dot{\gamma}(y)$ is homogeneous at the scale of the capsule and constant in the $z$ direction. Capsules were visualized from the side of the channel in the $z$ direction. The steady-state shape of the capsule in the plane $(x, y)$ is characterized by its semi axis length $(L, S)$ and its inclination angle $\alpha$ with respect to the flow direction. Thin arrows show the motion of rotation of the membrane during shear, the so-called tank-treading motion.

length (in $y$ direction) (figure 2) where the shear rate $\dot{\gamma}$ was maximal and the flow profile almost linear. Particle-tracking velocimetry in the ROI showed that the flow was planar, i.e. independent of the $z$ direction. As expected, the flow profile was parabolic in $y$ and constant in $x$ and $z$ whatever the flow rate of the pump. The $y$ position of the capsule was measured to determine accurately the local shear rate $\dot{\gamma}(y)$. For distance at the wall below $200 \mu \mathrm{m}$, the maximal variation of $\dot{\gamma}$ at the tips of the deformed capsule was less than $\pm 7.5 \%$. Thus the capsules are assumed to move in a constant shear rate flow in this region. $\dot{\gamma}$ ranged from 10 to $2000 \mathrm{~s}^{-1}$. Experiments were carried out at a room temperature of $22 \pm 0.5^{\circ} \mathrm{C}$ controlled locally. The viscosity of the glycerol $\eta_{f}$ was measured with a rheometer ThermoScientifc Mars III and ranged from 0.99 to 1.18 Pa.s.

To obtain high steady deformations, the capsule was initially put far from the ROI. The flow was then applied and the capsule crossed the area separating it from the ROI. The steady-state of the deformation was thus already reached, when the capsule arrived in the ROI. The capsule was observed during 5 to $100 \mathrm{~ms}$ according to the shear rate. The maximal velocity of the capsule was about $180 \mathrm{~mm} / \mathrm{s}$. The flow was stopped when the capsule was out of the ROI. The two axes of the ellipsoid $(L, S)$ and the angle of inclination $\alpha$ were measured (figure 2). We checked that these parameters of shape were steady and that the capsules returned to their initial spherical shapes at rest. The velocity of the capsule was also measured and compared with preliminaries PTV measurements to check that the presence of the walls had no effects: no difference is measured between a capsule velocity and its corresponding streamline without capsule measured by PTV. The direction of the capsule being parallel to the wall in the ROI, it means that the gradient of shear did not generate significant lateral migration on this short distance. Each of the twenty capsules was studied for approximately seven different shear rates. The inner and outer viscosities were equal. All the capsules which were not spherical (difference of two pixels between the large and small axis) were excluded. 

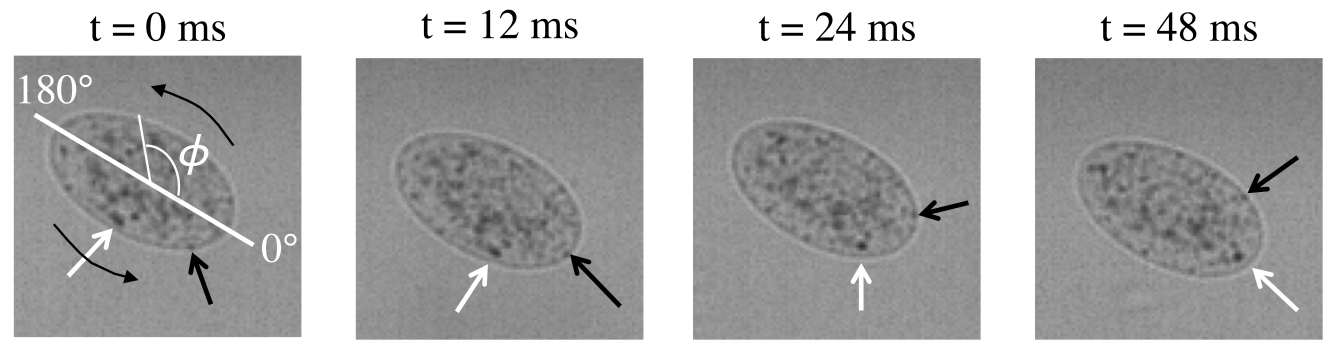

(a)

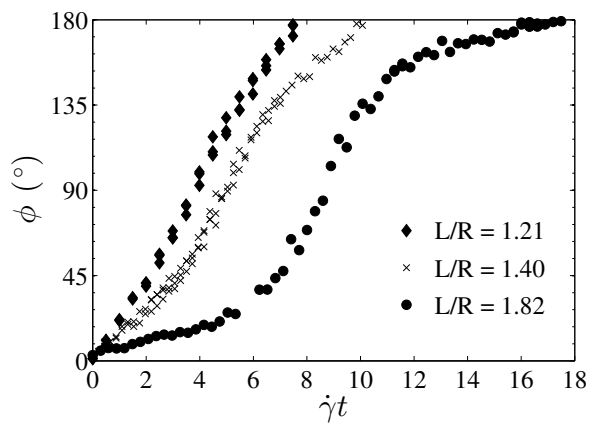

(b)

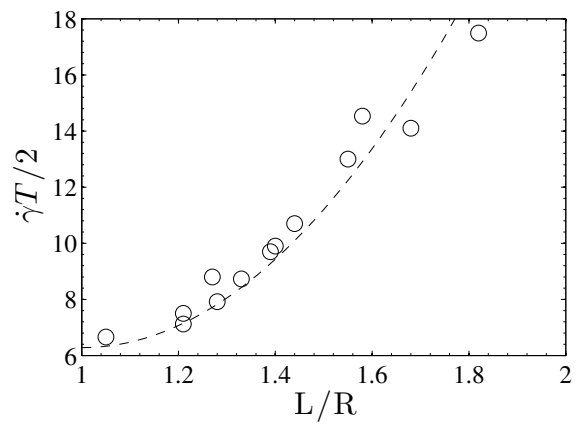

(c)

Figure 3: Tank-treading motion of the membrane of microcapsules. (a) iron oxide particles rotate with the membrane during shear flow. Different particles located at the periphery of a capsule (black and white arrows) were followed during a sequence to reconstruct the evolution of the angle $\phi$ with time $t$. (b) Angle of rotation $\phi$ as a function of the dimensionless time $\dot{\gamma} t$ for different stretching of the large axis $L / R$. (c) Half dimensionless time period of rotation $\dot{\gamma} T / 2 . \dot{\gamma} T / 2=2 \pi$ corresponds to the half period of rotation of a rigid sphere in shear flow. Dashed line: parabolic fit.

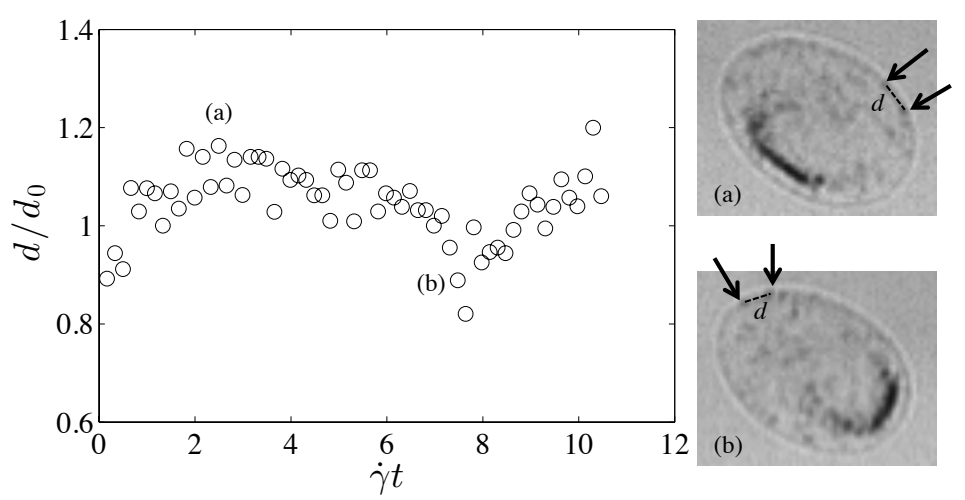

Figure 4: Local evolution of the deformation of the membrane: the relative distance $d / d_{0}$ between two closed iron oxide particles (at rest $d_{0}=14.8 \mu \mathrm{m}$ ) is plotted as a function of the dimensionless time $\dot{\gamma} t$ for $L / R=1.21$. Particles are located on the tips of the capsule for $\dot{\gamma} t=0$ and 7.5 and on its side for $\dot{\gamma} t=4$. 


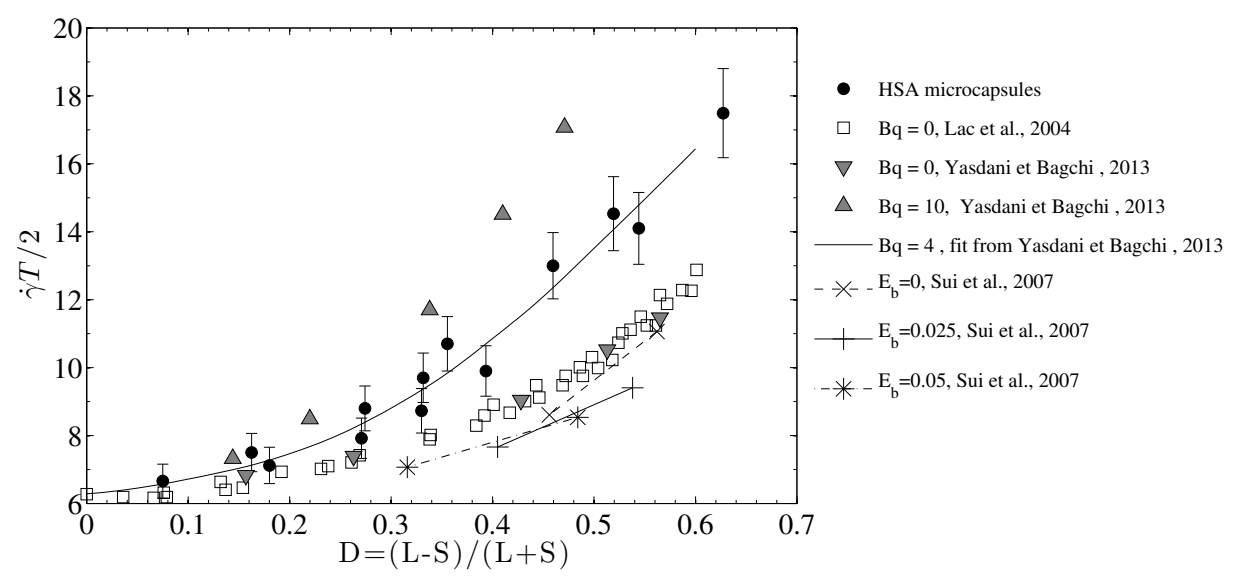

Figure 5: Tank-treading period as a function of the Taylor parameter $D$. The period increases with the membrane viscosity $\eta_{m}$. For $\eta_{m}=0$, the period is independent of the constitutive law: numerical results from Lac et al. (2004), Yazdani \& Bagchi (2013) and Sui et al. (2007). Conversely, the period decreases when the bending energy $E_{b}$ increases.

\section{Results and discussion}

\subsection{Tank-treading motion of the membrane}

In a simple shear flow, an initially spherical capsule becomes a steady ellipsoid with a constant angle of inclination $\alpha$ (figure 2) whatever the shear rate is. The instantaneous motion of the membrane, namely a rotation (see Videos in Supplementary Materials), was visualized by individual micro-particles embedded in the membrane (figure 3). Except at very low shear rate, the length of the ROI was too small to observe a full half-period of tank-treading. This last was reconstructed by tracking several particles. We checked that the shear elastic moduli of eight capsules were not changed by these micro-particles using de Loubens et al. (2014). The movement of rotation of the membrane during shear flow, referred as tank-treading, is dependent on the time $t$ and on capsule stretching $L / R$. For stretching lower than $20 \%$, the evolution of the angle of rotation $\phi$ with time is linear. When the stretching (or the shear rate) increases, this evolution deviates more and more from linearity. Whereas the velocity of rotation $(d \phi / d t)$ at $90^{\circ}$ of the tips is almost constant when $L / R$ increases, this velocity decreases strongly at the tips (figure $3-\mathrm{b}$ ). For small deformations $(L / R \leq 1.1)$, the period of rotation $\dot{\gamma} T$ corresponds to the case of a rigid sphere in shear flow. $\dot{\gamma} T$ versus $L / R$ is well fitted by a parabola. Quantitatively, the period triples when $L / R$ increases of $80 \%$.

The local deformations of the membrane could be derived from the evolution of the angle $\phi$ with time $t$. However the derivation is not robust due to the level of noise of these data. Measuring the time-evolution of the distance $d$ separating two particles is a better method (figure 4). We observe that the membrane deformation is inhomogeneous. The membrane is tensed at the tips and stretched on the sides. For an extension of $L / R=1.21$, the distance separating the particles increases of $\pm 20 \%$ between the side and the tips of the capsule. The difficulty of the experiment did not allow to repeat it on the same capsule for different shear rates with a sufficient accuracy to separate clearly the results but the trends are similar. 


\subsection{Evaluation of membrane viscosity from the tank-treading period}

The tank-treading motion is the basic dynamics of numerous soft particles. It involves a membrane shear flow contributing to a two-dimensional dissipation. Contrary to the steady state of a capsule in an elongation flow, its tank-treading motion in shear flow is a good candidate to reveal quite easily any membrane viscosity $\eta_{m}$. Indeed, numerical simulations (Lac et al. (2004)) show that the period is independent of the constitutive law which governs the elastic response of the membrane (figure 5). Our experimental results are determined in the regime of large deformations. They are also reported on the same figure highlighting a notable increasing of the tank-treading period. Any deviation from the numerical master curve should be explained by contributions to the interfacial mechanics which were neglected previously as the bending resistance or the membrane dissipation due to membrane viscosity.

Sui et al. (2007) have analyzed the effects of bending rigidity on the deformation $D$ and the tank-treading period: see figures 5 and 7 a of their paper. Their simulations reported on figure 5 showed that the bending modulus decreases slightly the tank-treading period $\dot{\gamma} T$, the opposite of what is observed experimentally.

Yazdani \& Bagchi (2013) have studied numerically the contribution of the shear membrane viscosity on tank-treading motion introducing the Boussinesq number $\mathrm{Bq}$ :

$$
B q=\frac{\eta_{m}}{\eta_{f} R}
$$

which measures the surface shear viscous stress compared to bulk one, $\eta_{m}$ and $\eta_{f}$ being the membrane and fluid viscosities respectively. They showed that the period increases with the Boussinesq number. Combining figures 11 and 13 of Yazdani \& Bagchi (2013) allows to report in figure 5 their results for $B q=0$ and $B q=10$, the smaller values of Boussinesq numbers which have been considered. The period increases with $B q$ keeping the same parabolic curve with the Taylor parameter. Our experimental data are included between the case $B q=0$ and $B q=10$ and have the same parabolic variation. In absence of more precise numerical simulations and to obtain an order of magnitude of $B q$ that fits our data, we assumed a linear relationship of the tank-treading period $\dot{\gamma} T / 2$ with $B q$. On a theoretical point of view, considering a constant ellipsoidal shape, Keller \& Skalak (1982) have shown that the period of tank-treading varies linearly with the membrane viscosity, validating our assumption to consider a linear fit. In this way, the best fit corresponds to $B q \approx 4: \eta_{m} \approx 2.10^{-4} \mathrm{~N} . \mathrm{s} / \mathrm{m}$ for $R=43 \mu \mathrm{m}$ for example. This value of $B q$ obtained in the regime of large deformations will be confirmed by a second independent method in the regime of moderate deformations (see section 3.5).

To show that our approach is self consistent, the membrane deformation and the viscoelastic time scales are compared. The first time scale is measured experimentally with the evolution of the local deformation of the membrane with the dimensionless time (figure 4) independently of any assumptions concerning the membrane properties. The dimensionless characteristic time of the local rate of membrane deformation is about $\dot{\gamma} t=2$ for medium deformation of the capsule $(L / R=1.21)$. In physical units, this time scale is about $50 \mathrm{~ms}$. It can be compared to the time scale for the viscoelastic membrane that is defined by $\eta_{m} / G_{s}$ and equal to $40 \mathrm{~ms}$ of the same order than previously.

\subsection{Shapes of microcapsules in shear flow}

In shear flow, HSA microcapsules sustain up to $115 \%$ of stretching of their large axis $L$ without burst. For stretching of $L$ higher than $5 \%$, two regimes of steady deformations are observed according to the magnitude of $L / R$. In the range $1.05<L / R<1.7$, the shape of the capsule is ellipsoidal (figure 6). Qualitatively, its length $L$ increases with the 


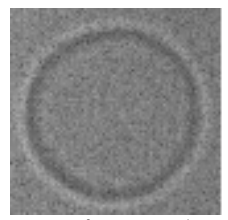

$\mathrm{L} / \mathrm{R}=1 \quad \mathrm{~L} / \mathrm{R}=1.50$

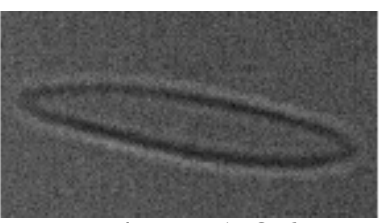

$\mathrm{L} / \mathrm{R}=1.96$

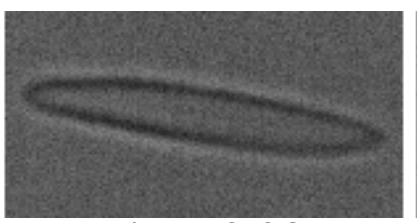

$\mathrm{L} / \mathrm{R}=2.08$

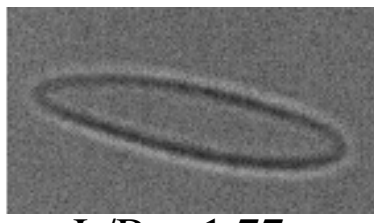

$\mathrm{L} / \mathrm{R}=1.77$

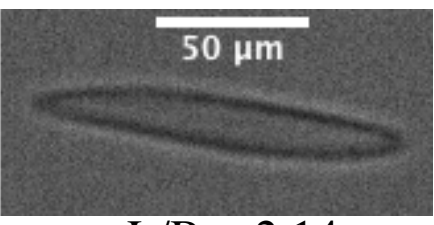

$\mathrm{L} / \mathrm{R}=2.14$

Figure 6: Typical evolution of the steady-state shape of a microcapsule $(R=28 \mu \mathrm{m})$ for increasing shear rates: the dimensionless capsule length $L / R$ varies up to $114 \%$. For moderate deformations $(L / R \leq 1.7)$, the capsule is ellipsoidal and symmetrical to the large and small axis. For larger deformations, the shape exhibits a S-like shape with the tips of the capsule oriented in the flow direction.
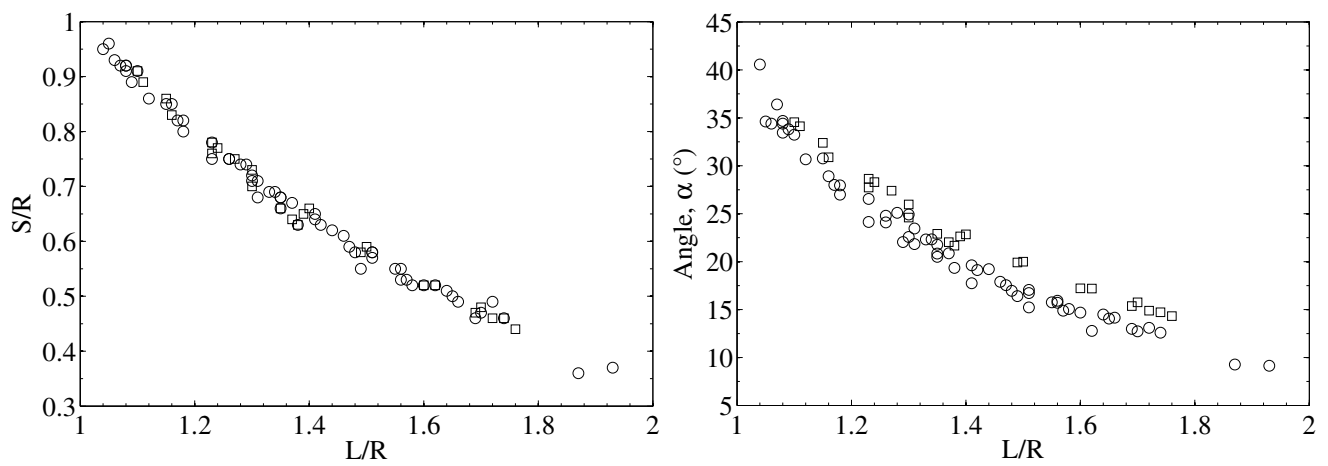

Figure 7: Experimental variation of the small length $S / R$ and the inclination angle $\alpha$ as a function of $L / R$ in shear flow for two different batches of microcapsules: ०: $20 \%$ HSA, $\square$ : $25 \%$ HSA. The radii of capsules vary from 25 to $60 \mu \mathrm{m}$ in each batch. Errors correspond approximately to $\pm 5 \%$ on $L / R$ and $S / R$ and $\pm 1.5^{\circ}$ on $\alpha$.

shear rate while its width $S$ decreases. For $L / R$ higher than 1.7, the shape looks like an 'S' whose the extremities are aligned with the flow direction (see video in supplementary materials). These S-like shapes are reminiscent of the transient ones obtained by Chang \& Olbricht (1993a) and Koleva \& Rehage (2012) for artificial capsules that bursted for minute deformations. However, it is notable that these shapes are steady for the HSA capsule of the present study, whereas it was unsteady during tips break-up of polysiloxane (Koleva \& Rehage (2012)) and nylon (Chang \& Olbricht (1993a)) capsules. These steady shapes have been also observed numerically by Walter et al. (2010) for deformations $((L-S) /(L+S) \approx 0.55)$ equivalent to those we report.

According to the simulations of these authors, compressive tensions appear at weak stretching in the center part of the capsule membrane and at large stretching near the tips. In each case, wrinkles are expected (Finken \& Seifert (2006)). Here and contrary 

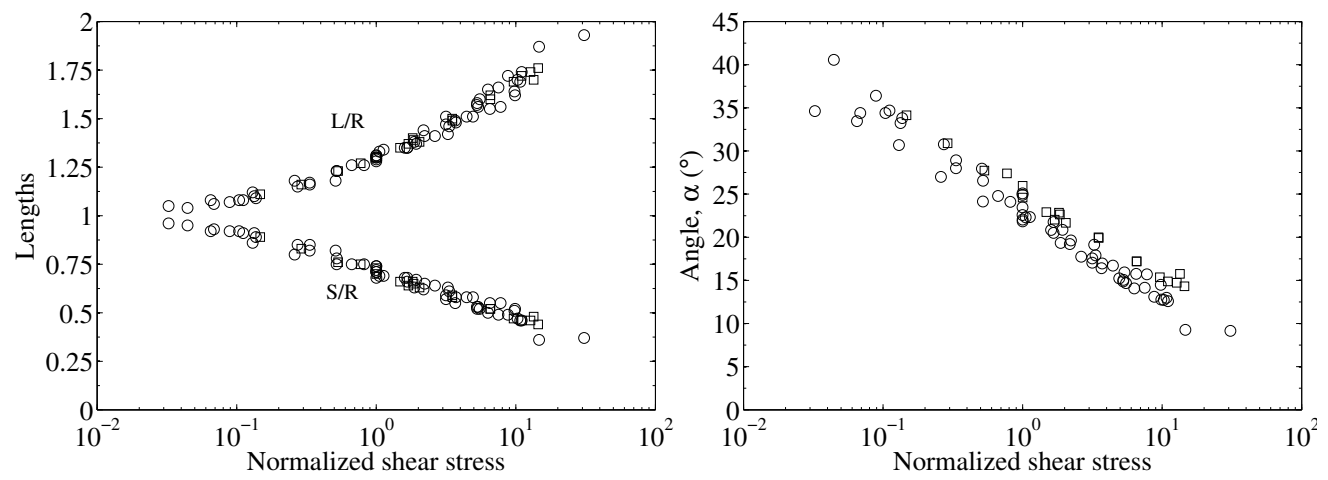

Figure 8: Deformations as a function of the viscous shear stress $\eta \dot{\gamma}$ : principal half-lengths of the capsule $S / R$ and $L / R$ (left) and inclination angle $\alpha$ (right). The shear stress $\eta \dot{\gamma}$ is normalized by its value in $L / R=1.3$. Errors on $\eta \dot{\gamma}$ are approximately $\pm 5 \%$. ०: $20 \% \mathrm{HSA}$, $\square: 25 \%$ HSA.

to well-known experiments with polysiloxane capsules (Walter et al. (2001), Koleva \& Rehage (2012)), no wrinkles are observed meaning that either the threshold for buckling is not reached due to a too large bending resistance or the time to develop such shape modulations is out of range with our set-up. A third possibility could be a weak optical contrast due to fast imaging. However, firstly, even at small shear rates, there are no visible wrinkles. Secondly, the same report of no wrinkling was done with the same capsules in an elongational flow (de Loubens et al. (2015)). This supports that bending resistance inhibited the localized shape modulations at short wavelength, i.e wrinkles.

Capsules with two HSA concentrations and with radii in the range 20 to $68 \mu \mathrm{m}$ were studied. It is noteworthy that the variations of the width $S / R$ and the inclination angle $\alpha$ with the longest axis $L / R$ are independent of the kind of capsules with all the experimental points on two master curves: figure 7. The inclination angle $\alpha$ of the capsule decreases with the flow from $40^{\circ}$ to $8^{\circ}$ for $L / R$ ranging from 1.05 to 2 . The capsule tends to be aligned with the flow as expected (Lac et al. (2004)). This has already been observed experimentally for vesicles (Kantsler \& Steinberg (2005)) and for drops (Guido \& Villone (1998), Kennedy et al. (1994)). The inclination angle of $45^{\circ}$ corresponds to the elongation part of the simple shear flow, a result predicted for drops a long time ago in the seminal article of Taylor (1934).

\subsection{Deformations with the viscous shear stress and the Capillary number}

The previous analysis was purely geometrical. In this section, we introduce firstly the effects of the viscous shear stress $\eta \dot{\gamma}$. We normalized the shear stress $\eta \dot{\gamma}$ by a value of reference at $L / R=1.3$ for each capsule in the regime of moderate deformations: figure8. The conclusions are independent of this choice. All the parameters of shape $(L / R, S / R$ and $\alpha$ ) follow the same master curves on three orders of magnitude of variation of $\eta \dot{\gamma}$ whatever the size and the degree of cross-linking of the capsules are. The inclination angle $\alpha$ decreases exponentially with $\eta \dot{\gamma}$ (plot in semi-log) in qualitative agreement with numerical simulations (Lac et al. (2004)). The increase (decrease) of $L / R(S / R)$ is more and more moderate when the shear stress increases. The shear stress has to increase by an order of magnitude to stretch the capsule from 50 to $100 \%$. The capsule being more and more aligned with the flow direction when $\eta \dot{\gamma}$ increases, the elongation component of the simple shear flow (maximal at $45^{\circ}$ ) has less and less effects on the capsule deformation. 


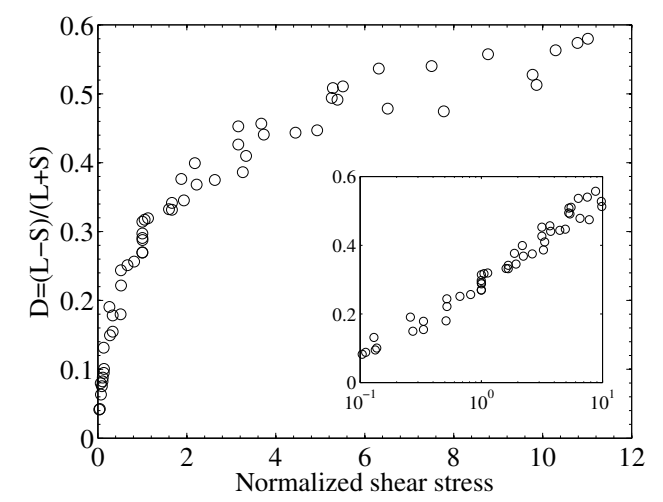

Figure 9: Taylor parameter $D=(L-S) /(L+S)$ as a function of the viscous shear stress $\eta \dot{\gamma}$. Insert: semi-log representation.
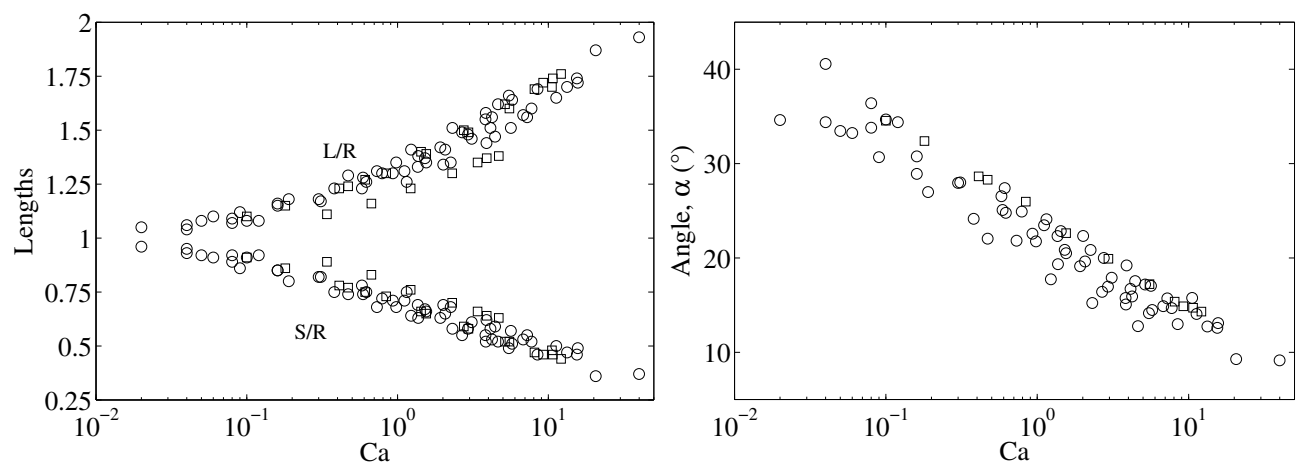

Figure 10: Deformations as a function of the Capillary Number $C a=\eta \dot{\gamma} R / G_{s}$ : principal half-lengths of the capsule $S / R$ and $L / R$ (left) and inclination angle $\alpha$ (right). Errors on $C a$ are approximately $\pm 20 \%$. ॰: $20 \%$ HSA, $\square: 25 \%$ HSA.

The Taylor parameter $D=(L-S) /(L+S)$ of all the studied capsules follows also a master curve which has the characteristic to not saturate at high normalized shear stress (figure 9) in qualitative agreement with numerical simulations (Lac et al. (2004)).

After showing that the data can be scaled by an arbitrary shear stress of reference, we scale them also with the surface shear elastic modulus $G_{s}$ (in N/m) calculated from the fits shown in figures 1 . The capillary number $C a$ is defined as the ratio of the hydrodynamic viscous shear stress $\eta \dot{\gamma}$ by the elastic response $G_{s} / R$ of the membrane:

$$
C a=\frac{\eta \dot{\gamma} R}{G_{s}}
$$

Note that we use the shear elastic modulus and not the surface Young modulus as Barthes-Biesel \& Sgaier (1985): see the next section. The evolutions of each parameter of shape $L / R, S / R, \alpha$ and $D$ follow four master curves on more than three orders of magnitude of variation of $C a$ whatever the size and the degree of cross-linking of the capsules: figures 10 and 11. The uncertainties on the regressions $G_{s}=f(R)$ (errors on $C a$ are $\pm 20 \%$ ) provide a larger dispersion of the experimental data as a function of $\mathrm{Ca}$ compared to the normalized shear stress $\eta \dot{\gamma}$ (figure 8). These evolutions can be compared 


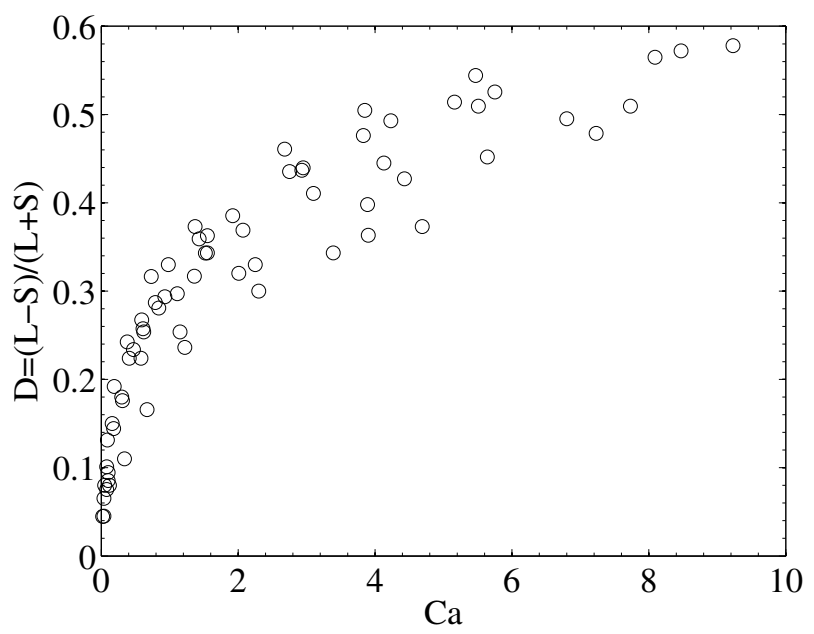

Figure 11: Taylor parameter $D=(L-S) /(L+S)$ as a function of the Capillary Number $C a=\eta \dot{\gamma} R / G_{s}$.

with previous numerical simulations. Dupont et al. (2015) simulated the evolution of $D$ with $C a$ (see figure $6 \mathrm{~b}$ of their paper) for the constitutive law corresponding to HSA capsules, i.e. the generalised Hooke law (de Loubens et al. (2015)). Numerical and experimental curves are qualitatively correct, but there are large quantitative differences. For a value of $C a$ of 2 , a Taylor parameter $D$ of 0.55 is numerically calculated, whereas we obtained experimentally 0.3 . These differences could be attributed to the absence of membrane viscosity in the simulations of Dupont et al. (2015): see the next section.

\subsection{Evaluation of membrane viscosity from small deformation theory}

Barthes-Biesel \& Sgaier (1985) have determined the theoretical variation of the Taylor parameter $D=(L-B) /(L+B)$ as a function of the capillary number $C a$ and the Boussinesq number $B q$. Their main assumptions were to consider an incompressible 3D material of membrane leading to $\nu_{s}=1 / 2$, deformations such as $D<<1$ to expand the physical quantities and equal dilational and shear membrane viscosities. Note that this last assumption is different from the work of Yazdani \& Bagchi (2013) who have neglected the effect of dilational viscosity. This will be discussed in the conclusion. For small capillary number and without membrane viscosity, the relation is:

$$
D=\frac{25}{12} C a
$$

Note that there is a coefficient $1 / 3$ with the expression of Barthes-Biesel \& Sgaier (1985). This comes from a different definition of the capillary number. Indeed, Barthes-Biesel \& Sgaier (1985) used the surface Young modulus $E_{s}$ while we use the shear elastic modulus which satisfies: $G_{s}=(1 / 3) E_{s}$. Currently, it is more usual to consider the shear elastic modulus at least in the field of capsules. The ratio of characteristic elastic $\eta R / G_{s}$ and surface viscous $\eta_{s} \dot{\gamma} / \eta R$ times introduces a new dimensionless number $\beta$ which is analog to the Weissenberg number in viscoelastic fluids:

$$
\beta=\frac{\eta_{s} \dot{\gamma}}{G_{s}}=B q C a
$$

Here also, the quantity $\beta$ is three times larger than the definition of Barthes-Biesel \& 


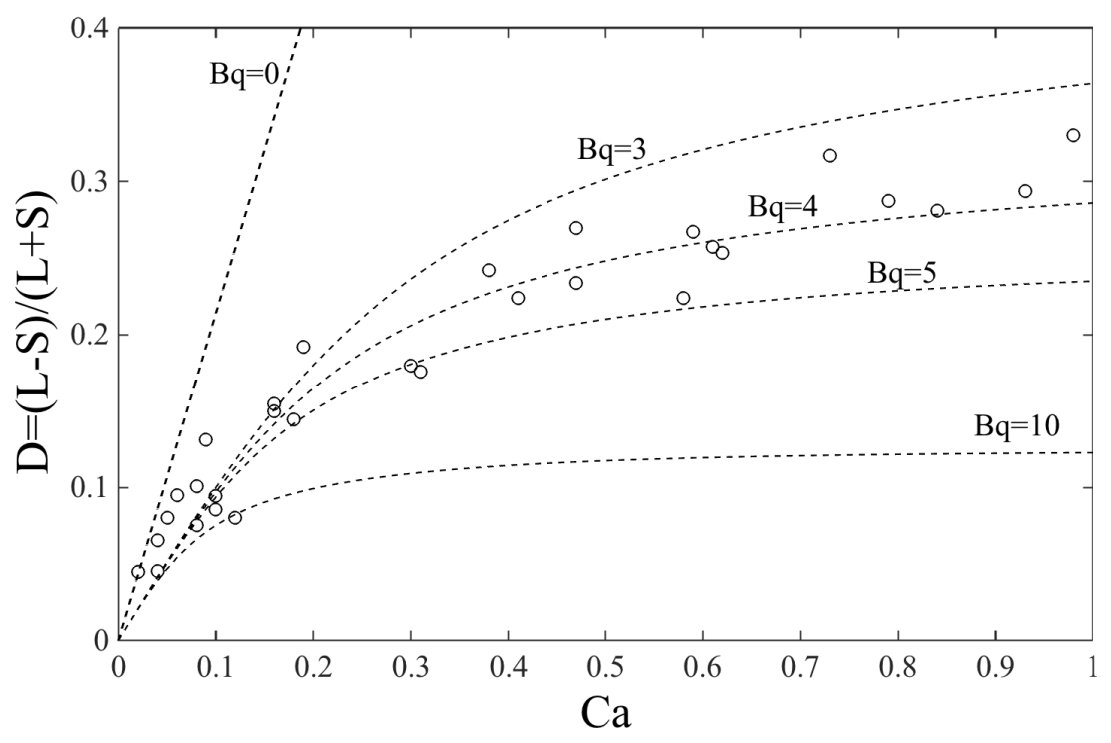

Figure 12: Left - Comparison between theoretical prediction (Barthes-Biesel \& Sgaier 1985) and our experimental results of the Taylor parameter in the limit of capillary number $C a<1$. The dashed lines are theoretical curves for several Boussinesq numbers $B q=\eta_{m} / R \eta_{f}$ equal to $3,4,5$ and 10 (equation 3.5) and 0 (equation 3.3).

Sgaier (1985) for the same reason than previously. Thus, in the limit of small capillary number and moderate value of Weissenberg number $(\beta=O(1))$, the relation becomes:

$$
D=\frac{25}{12} C a\left(\frac{1+\left(\frac{2 \beta}{5}\right)^{2}}{\left(1+\beta^{2}\right)\left(1+\left(\frac{\beta}{3}\right)^{2}\right)}\right)^{1 / 2}
$$

Note that the equation 3.3 is the limit of equation 3.5 when $\beta$ tends to zero. The experimental points for which the shape is stationary are compared to the two previous equations in figure 12. For very small capillary number $C a<0.15$ (or $D<0.1$ ), the dynamics of capsules depends crucially on the initial asphericity even if very small (see the next section). The experimental data with $D<0.1$ in figure 12 correspond to steady state shapes but are obtained only for the largest capsules $(R \approx 55 \mu \mathrm{m})$. If we consider larger capillary numbers, the deformations are restricted to $D<0.32$ providing a range of moderate deformations from $C a=0.15$ to $C a=1$ with steady state shapes. The theoretical curves $D=f(\mathrm{Ca})$ provided by equations 3.3 and 3.5 have been drawn for several Boussinesq numbers $B q$ equal to $0,3,4,5$ and 10 . For $C a>0.15$, the experimental points are scattered between $B q=3$ and $B q=5$ with a best fit $B q \approx 4$ on all the range of capillary number in figure 12. It is noteworthy that it corresponds to the same value determined by the analysis of the period of capsules using the numerical results of Yazdani \& Bagchi (2013) in section 3.2. These two independent methods support the fact that HSA membranes of microcapsules have a shear membrane viscosity.

Moreover, as the Boussinesq number depends on the size capsule, it means that the shear membrane viscosity increases approximately linearly with the radius of the capsule at least in the range $30-60 \mu \mathrm{m}$ considered in this paper: $\eta_{m}=1.3 \mathrm{~N} . \mathrm{s} / \mathrm{m}$ for $R=32.5 \mu \mathrm{m}$ and $\eta_{m}=2.5 \mathrm{~N} . \mathrm{s} / \mathrm{m}$ for $R=60 \mu \mathrm{m}$. The explanation is the same than for the shear elastic modulus (de Loubens et al. $(2014,2015)$ ). This is due to the process of fabrication 
(interfacial polymerization in batch) and the fact that approximately all HSA proteins inside the initial droplet polymerize at the interface. The error on the shear membrane viscosity is approximately $20-25 \%$ due to the error on the determination of the capillary number. This poor accuracy does not allow to conclude clearly on the effect of initial HSA concentration on the membrane viscosity. We expected to a rising of $\eta_{m}$ with HSA rate.

\subsection{Swinging in small deformations}

In the regime of small deformations (i.e. $D$ smaller than 0.1 ), we observed shape oscillation phenomena whereas the deviation from the sphericity of the capsule at rest was barely optically resolved. Note that among the largest microcapsules, some of them didn't exhibit oscillations for $0.04<D<0.1$. An example for a capsule of mean radius of $44 \pm 1 \mu \mathrm{m}$ with an initial shape asymmetry which can be characterized by an initial Taylor parameter $D_{0}$ equal to 0.012 is shown in figure 13 . The effects of this small deviation from the sphericity has notably dramatic effects on the amplitude of the orientation angle at small shear rate. The amplitude is of about $\pm 20^{\circ}$ for shear rate of $0.53 \mathrm{~s}^{-1}$ and decreases when the shear rate increases. The frequencies of oscillation for $\alpha$ and $D$ are equal with a phase shift of $\pi / 2$. The mean amplitude of $D$ is constant. The frequency and the amplitude of oscillations of $D$ increase with the shear flow, a result reminiscent of recent theoretical results (Kessler et al. (2009), Vlahovska et al. (2011)). The oscillation period is about $2 \pi / \dot{\gamma}$. This known motion of soft particles was called swinging (Vlahovska et al. (2009), Abreu et al. (2014)). Swinging has been reported for polyamide microcapsules by Walter et al. (2001) with qualitative features similar to those described for HSA microcapsules. We confirmed then their conclusion that the slightest deviation from sphericity is sufficient to induce swinging motion. Small-deformation theory has addressed only the spherical shape, but swinging was predicted by numerical simulations of Ramanujan \& Pozrikidis (1998) for nearly spherical shapes ( $L / S$ at rest of 0.9). Again, our observations are consistent with these numerical simulations. However the transitions between swinging, tank-treading and even tumbling (Kessler et al. (2008)) would benefit from deeper and systematic analysis if we were able to produce microcapsules with a controlled asphericity and constant membrane thickness. Note that swinging is also an easy mean to select spherical capsules. Readers should note that swinging motion was negligible for all the data reported in this paper (i.e. amplitude of oscillation inferior to $1 \%$ of the mean signal), expected in this section.

\section{Conclusion}

The behavior of microcapsules in weak to strong shear flow has been experimentally investigated with a large range of deformations and variation of the inclination angle over three decades of shear stress. Different phenomena were observed: tank-treading motion with the visualisation of membrane rotation, S-shapes for large deformations and swinging due to small deviation from the sphericity. No wrinkles were observed contrary to experiments with polysiloxane capsules (Walter et al. (2001), Koleva \& Rehage (2012)) meaning that bending resistance inhibited the localized shape modulations at short wavelength. According to the magnitude of the bending resistance, wrinkles formation can be prevented, but without significant effects on the shape and the inclination angle of the capsule (Yazdani \& Bagchi (2013), Dupont et al. (2015)). At this stage, we cannot conclude that bending resistance has a significant impact on shape and dynamics of HSA microcapsules. This conclusion is also supported by two other observations. In elongation flow de Loubens et al. (2015), situation for which a role of viscous dissipation 

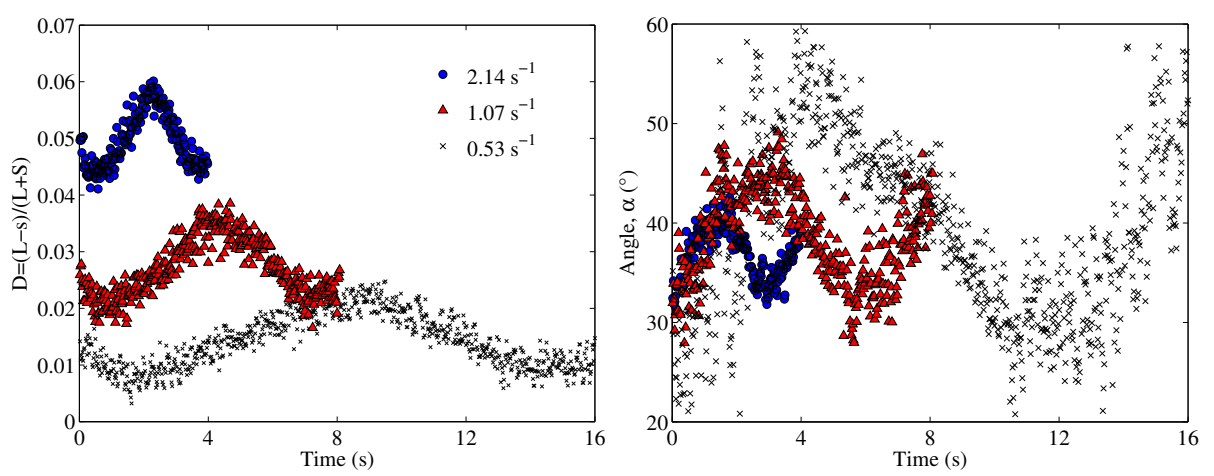

Figure 13: Swinging of microcapsules in the small deformation regime: the Taylor parameter $D$ and the inclination angle $\alpha$ oscillates with a frequency proportional to the shear rate. Example taken for a capsule of $44 \mu \mathrm{m}$ radius with an initial asymmetry $D_{0}$ equal to 0.012 at rest.

in the membrane is precluded for the stationary shape, the results of $2 \mathrm{D}$ elastic models of membrane and the experimental data were in very good agreement without the need to introduce bending effects. Also, numerical simulations of Pozrikidis (2001) show that the tips are more and more rounded when the bending stiffness increases. 'S-like' shapes with pointed tips can then be considered as a signature of absence of sufficient bending effects to affect the global shape of the capsule.

Moreover, our study supports a contribution of membrane viscous dissipation to explain the characteristics of the tank-treading motion of HSA microcapsules. Firstly, the variation of the dimensionless period of tank-treading $\dot{\gamma} T$ with the Taylor parameter $D$ was compared to numerical results of literature in the regime of large deformations: figure 5. This framework presents a crucial interest for the analysis because the results are independent of the choice of the $2 \mathrm{D}$ elastic constitutive law of the membrane. As bending resistance and membrane dissipation have opposite effects, the result can only been explained by a shear membrane viscosity. Secondly and probably the more convincing argument, in the regime of small capillary number $\mathrm{Ca}$ and moderate deformations (only swinging in the limit of very small deformations), the experimental variation of the Taylor parameter with $C a$ was compared to the analytical result of Barthes-Biesel \& Sgaier (1985) which takes into account dilational and shear membrane viscosities with equal weights. Experimental results deviate clearly from the case with no membrane dissipation. It is noteworthy that the same Boussinesq number was determined by these two independent methods in large and moderate regimes of deformations: $B q \approx 4$ which leads to $\eta_{m} \approx 1-210^{-4} \mathrm{~N} . \mathrm{s} / \mathrm{m}$ for capsule radius in the range $30-60 \mu \mathrm{m}$. The membrane viscosity varies approximately linearly with the radius of microcapsules in this range due to the process of fabrication. However, further studies with a large range of radius up to several tenths of micrometers will be necessary to investigate fully how the membrane viscosity varies with the radius of capsules and also with the initial HSA concentration.

Finally, the result of the Boussinesq number which is the same with the two methods questions also the relative contributions of dilational and shear membrane viscosities. A first response could be that in shear flow, the shape is dominated by contribution of the shear membrane viscosity due to the rotation of the surface, a result recently observed numerically in the case of droplets with both surface viscosities in shear flow (Gounley et al. (2015)). Thus, to be optimal, the numerical simulations and models should consider 
various constitutive laws and notably the generalized Hooke model for HSA microcapsules (de Loubens et al. (2015)), the bending resistance and the dilational and shear membrane viscosities which is not the case currently.

This work has benefited of financial support from the ANR CAPSHYDR (11-BS09013-02), from the ANR Polytransflow (ANR-13-BS09-0015-01), from Labex MEC (ANR11-LABX-0092), from A*MIDEX (ANR-11-IDEX-0001-02) and from CNES.

\section{REFERENCES}

Abkarian, M., Faivre, M. \& Viallat, A. 2007 Swinging of red blood cells under shear flow. Physical review letters 98, 188302.

Abreu, D., Levant, M., Steinberg, V. \& Seifert, U. 2014 Fluid vesicles in flow. Adv. Colloid Interf. Sci. 208, 129-141.

Andry, M. C., Edwards-Levy, F. \& Levy, M. C. 1996 Free amino group content of serum albumin microcapsules. iii. a study at low ph values. Int. J. Pharm. 128, 197-202.

Bagchi, P. \& Kalluri, R. M. 2009 Dynamics of nonspherical capsules in shear flow. Phys. Rev. E 80, 016307.

BARThÈs-Biesel, D. 2011 Modeling the motion of capsules in flow. Curr. Opin. Colloid Interface Sci. 16, 3-12.

Barthes-Biesel, D \& Sgaier, H 1985 Role of membrane viscosity in the orientation and deformation of a spherical capsule suspended in shear flow. Journal of Fluid Mechanics 160 (119-135).

Chang, K. S. \& Olbricht, W. $1993 a$ Experimental studies of the deformation and breakup of a synthetic capsule in steady and unsteady simple shear flow. J. Fluid Mech. 250, 609-633.

Chang, K. S. \& Olbricht, W. $1993 b$ Experimental studies of the deformation of a synthetic capsule in extensional flow. J. Fluid Mech. 250, 587-608.

Dodson, W. R. III \& Dimitrakopoulos, P. 2009 Dynamics of strain-hardening and strainsoftening capsules in strong planar extensional flows via an interfacial spectral boundary element algorithm for elastic membranes. J. Fluid Mech. 641, 263-296.

Dupont, C, Salsac, A-V, Barthès-Biesel, D, Vidrascu, M \& Le Tallec, P 2015 Influence of bending resistance on the dynamics of a spherical capsule in shear flow. Physics of Fluids 27 (5), 051902.

Finken, R. \& Seifert, U. 2006 Wrinkling of microcapsules in shear flow. J. Phys. Condens. Matter 18, L185-L191.

Fisher, T. M., Stohr-Liesen, M. \& Schmid-Schonbein, H. 1978 The red cell as a fluid droplet: tank tread-like motion of the human erythrocyte membrane in shear flow. Science 202, 894-896.

Freund, J. B. 2014 Numerical simulation of flowing blood cells. Ann. Rev. Fluid Mech. 46, 67-95.

Gounley, J., Boedec, G., JAeger, M. \& Leonetti, M. 2015 Influence of surface viscosity on droplets in shear flow. submitted .

Guido, S. \& Villone, M. 1998 Three-dimensional shape of a drop under simple shear flow. Journal of rheology 42, 395-415.

Gunes, D. Z., Pouzot, M., Ulrich, S. \& Mezzenga, R. 2011 Tuneable thickness barriers for composite o/w and w/o capsules, films, and their decoration with particles. Soft Matter 7, 9206-9215.

Hejazi, Radi \& Amisi, Mansoor 2003 Chitosan-based gastrointestinal delivery systems. Journal of controlled release $\mathbf{8 9}$ (2), 151-165.

Hochmuth, R. M., Worthy, P. R. \& Evans, E. A. 1979 Red cell extensional recovery and the determination of membrane viscosity. Biophysical Journal 26, 101-114.

Kantsler, Vasiliy \& Steinberg, Victor 2005 Orientation and dynamics of a vesicle in tanktreading motion in shear flow. Physical review letters 95 (25), 258101.

Keller, S. R. \& SkAlak, R. 1982 Motion of a tank-treading ellipsoidal particle in a shear flow. Journal of Fluid Mechanics 120, 27-47. 
Kennedy, M. R., Pozrikidis, C. \& Skalak, R. 1994 Motion and deformation of liquid drops, and the rheology of dilute emulsions in simple shear flow. Computers Fluids 23, 251-278.

Kessler, S., Finken, R. \& Seifert, U. 2008 Swinging and tumbling of elastic capsules in shear flow. J. Fluid Mech. 605, 207-226.

Kessler, S., Finken, R. \& Seifert, U. 2009 Elastic capsules in shear flow: analytical solutions for constant and time-dependent shear rates. The European Physical Journal E 29, 339413.

Koleva, I. \& Rehage, H. 2012 Deformation and orientation dynamics of polysiloxane microcapsules in liner shear flow. Soft Matter 8, 3681-3693.

Lac, E., Barthès-Biesel, D., Pelekasis, N. \& Tsamopoulos, J. 2004 Spherical capsules in three-dimensional unbounded stokes flows: effect of the membrane constitutive law and onset of buckling. J. Fluid Mech. 516, 303-334.

Lefebvre, Y., Leclerc, E., Barthès-Biesel, D., Walter, J. \& Edwards-Lévy, F. 2008 Flow of artificial microcapsules in microfluidic channels: A method for determining the elastic properties of the membrane. Phys. Fluids 20, 123102.

Li, X., Vlahovska, P. M. \& Karniadakis, G. E. 2013 Continuum- and particle-based modeling of shapes and dynamics of red blood cells in health and disease. Soft Matter 9, 28-37.

Long, Yue, Liu, Chuanyong, Zhao, Bin, Song, Kai, Yang, Guoqiang \& Tung, Chen-Ho 2015 Bio-inspired controlled release through compression-relaxation cycles of microcapsules. NPG Asia Materials 7 (1), e148.

de Loubens, C., Deschamps, J., Boedec, G., \& Leonetti, M. 2015 Stretching of capsules in an elongation flow, a route to constitutive law. Journal of Fluid Mechanics 767, R3.

de Loubens, C., Deschamps, J., Georgelin, M., Charrier, A., Edwards-Lévy, F. \& LEONETTI, M. 2014 Mechanical characterization of cross-linked serum albumin microcapsules. Soft matter 10, 4561-4568.

Nash, G. B. \& Meiselman, H. J. 1983 Red cell and ghost viscoelasticity. Biophysical journal 43, 63-73.

Omori, T., Imai, Y., Yamaguchi, T. \& Ishikiwa, T. 2012 Reorientation of a nonspherical capsule in creeping shear flow. Phys. Rev. Lett. 108, 138102.

PozRIKIDIS, C. 2001 Effect of membrane bending stiffness on the deformation of capsules in simple shear flow. Journal of Fluid Mechanics 440, 269-291.

Ramanujan, S \& PozRikidis, C 1998 Deformation of liquid capsules enclosed by elastic membranes in simple shear flow: large deformations and the effect of fluid viscosities. Journal of Fluid Mechanics 361, 117-143.

Risso, F., Colle-Paillot, F. \& Zagzoule, M. 2006 Experimental investigation of a bioartificial capsule flowing in a narrow tube. J. Fluid Mech. 547, 149-173.

Shewan, Heather M \& Stokes, JAson R 2013 Review of techniques to manufacture microhydrogel particles for the food industry and their applications. Journal of Food Engineering 119 (4), 781-792.

Sui, Y, Chew, YT, Roy, P, Chen, XB \& Low, HT 2007 Transient deformation of elastic capsules in shear flow: effect of membrane bending stiffness. Physical Review E $\mathbf{7 5}$ (6), 066301.

Sui, Y., Low, H. T., Chew, Y. T. \& Roy, P. 2008 Tank-treading, swinging and tumbling of liquid-filled elastic capsules in shear flow. Physical Review E $\mathbf{7 7}, 016310$.

TAYlOR, G. I. 1934 The formation of emulsions in definable fields of flow. Proc. R. Soc. Lond. A 146, 501-523.

Vlahovska, P., Podgorski, T. \& Misbah, C. 2009 Vesicles and red blood cells in flow: From individual dynamics to rheology. C. R. Physique 10, 775-789.

Vlahovska, P. M., Young, Y.-N., Danker, G. \& Misbah, C. 2011 Dynamics of a nonspherical microcapsule with incompressible interface in shear flow. J. Fluid Mech.678, $221-247$.

Walter, A., Rehage, H. \& Leonhard, H. 2001 Shear induced deformation of microcapsules: shape oscillations and membrane folding. Colloid Suf. A 123, 183-185.

Walter, J., Salsac, A-V, Barthès-Biesel, D. \& Le Tallec, P. 2010 Coupling of finite element and boundary integral methods for a capsule in a stokes flow. Int. J. Numer. Meth. Engng. 83, 829-850. 
YAZDANi, A. \& BAGChI, P. 2013 Influence of membrane viscosity on capsule dynamics in shear flow. Journal of Flluid Mechanics 718, 569-595.

Zhu, L. \& BRANDT, L. 2015 The motion of a deforming capsule through a corner. Journal of Fluid Mechanics 770, 374-397. 